\title{
Meules et matériel de broyage (Préhistoire)
}

\section{Roubet}

\section{OpenEdition}

Journals

Édition électronique

URL : http://journals.openedition.org/encyclopedieberbere/585

DOI : $10.4000 /$ encyclopedieberbere.585

ISSN : 2262-7197

\section{Éditeur}

Peeters Publishers

\section{Édition imprimée}

Date de publication : 30 décembre 2010

Pagination : 4947-4954

ISBN : 978-90-429-2368-3

ISSN : 1015-7344

\section{Référence électronique}

C. Roubet, « Meules et matériel de broyage (Préhistoire) », Encyclopédie berbère [En ligne], 31 | 2010, document M101a, mis en ligne le 08 octobre 2020, consulté le 12 octobre 2020. URL : http://

journals.openedition.org/encyclopedieberbere/585 ; DOI : https://doi.org/10.4000/ encyclopedieberbere.585

Ce document a été généré automatiquement le 12 octobre 2020.

(c) Tous droits réservés 


\title{
Meules et matériel de broyage (Préhistoire)
}

\author{
C. Roubet
}

1 Les activités de broyage s. l. remonteraient en Afrique du Nord au Pléistocène final ; elles paraîtraient n'avoir été que circonstancielles et symboliques, lorsque les Hommes de Mechta-Afalou s'installent, il y a plus de 20.000 ans, sur le pourtour méditerranéen. Dans le mobilier, ce sont d'abord des molettes qui évoquent ces activités, mais pas encore des meules, plutôt des billots-enclumes naturels, sélectionnés et transportés, qui acquièrent alors le statut d'instruments intentionnels, après usage, en raison des traces diverses qu'ils conservent: incisions, martelages, colorants. Durant l'Holocène, on aurait tendance à considérer le domaine d'activités que recouvre ce matériel, comme plus ouvert, plus diversifié, et même encore compatible avec une relative mobilité des individus, en raison d'un apparent développement de cet équipement.

2 Il est habituel d'attribuer à cet équipement une double vocation. Aux fonctions antérieurement symboliques longtemps pratiquées, s'ajoutent celles d'une subsistance occasionnelle et saisonnière. Au Maghreb, à l'exception d'un cas marocain de production de céréales, qui sera présenté plus loin, l'alimentation végétale n'est pas issue d'une mise en culture, elle résulte de collectes; les traces bio-archéologiques n'ont pas été découvertes et manquent (déflation) dans la plus part des cas, ce qui n'autorise pas à dépasser le stade pré-agricole.

3 Au Sahara algérien et selon les régions, durant l'Holocène, molettes, broyeurs, pilons, mortiers, sont d'abord rattachés à des contextes anté-néolithiques. Plus tard, cet équipement se diversifie et prolifère dans les zones d'erg, sans bénéficier de l'appui d'un contexte végétal conservé. La précocité probable d'une Néolithisation initiale du faciès saharo soudanais* se fonde sur de probables productions de mil*, d'après $G$. Camps (1968) et l'argumentation confortant cette hypothèse est tirée du développement d'une vaisselle céramique sub-sphérique.

Le répertoire morphologique rendant compte de cet équipement préhistorique n'est pas encore satisfaisant. Les normes que l'on a tenté d'établir s'appuient sur la reconnaissance de critères intentionnels techno-typologiques, issus de transformations 
créées et acquises par l'usage, durant l'Holocène, c.-à-d., lorsque de constantes activités avaient déjà permis d'en définir les formes pour stabiliser les actions et les résultats (Roubet 1973). Cette démarche non diachronique, non fonctionnelle, n'a pas pu bénéficier d'un nombre suffisant de documents, issus de contextes chronologiquement définis. Ensuite, à l'exception de quelques expériences (Roubet 1979, p. 442, Fig. 201), aucune expérimentation systématique n'a été pratiquée, pour essayer de préciser les fonctions et la nature des produits obtenus. Ce domaine d'étude reste donc inexploité. A l'avenir, une nouvelle approche devrait permettre de mieux appréhender l'importance du développement de ce comportement, à finalité multiple, mais alimentaire dominante, très tardivement instauré au Maghreb. Les outils préhistoriques qualifiés de meule et de molette devraient avoir présenté avant usage ces caractéristiques :

5 Meule: lourd instrument lithique, transportable ou statique (en roches diverses, non tendres), dont l'assise pourrait être naturelle, incluse dans la roche ou bien celle d'un bloc aplani, préformé, présentant des témoins d'aménagements. La face opposée destinée au travail de broyage devrait être plane, ou nettement concave (mortier), puis avoir été régulièrement bouchardée et piquetée afin d'acquérir des caractéristiques de rugosité et de mordant, favorables au morcellement, à l'émiettement, à l'écrasement.

6 Molette : instrument maniable, dynamique, obtenu à partir d'un petit galet de roches résistantes, présentant naturellement deux faces au moins. L'une, destinée au broyage, devrait présenter les mêmes caractéristiques de mordant, pour que la substance placée entre la meule et la molette s'écrase et se réduise durant l'exercice de va et vient. L'autre, restée naturelle ou lisse, devrait faciliter la préhension à une ou à deux mains.

7 Lorsqu'on examine des documents de broyage provenant du contexte kubbaniyen du Paléolithique final de l'Egypte saharienne (vers $20000 \mathrm{cal} \mathrm{BC}$ ), on note d'abord le caractère semi-standardisé de ce matériel. En effet, vers Assouan, en bordure de grandes lagunes d'inondation, parallèles aux berges du Nil, plusieurs campements saisonniers ont livré une industrie épipaléolithique, voisine de l'Ibéromaurusien, associée à un équipement de broyage normé et diversifié, composé de meules, mortiers, broyeurs, molettes (Roubet 1989a); un atelier de fabrication de meules spécifiques a fait l'objet d'une étude du processus de création (Roubet 1989b). Si des habitudes de broyage se mettent en place très tôt en Egypte pré-néolithique, elles ne sont associées qu'à des collectes de végétaux sauvages. Ces données archéobotaniques se modifient un peu durant l'Holocène à Nabta Playa. Sorgho (Sorghum bicolor) et millets sauvages (Panicum turgidum et Echinochloa colona), sont parfois presents "only limited evidence of edible plant remains has been recovered, yet the presence of numerous grinding stones, as well as the limited plant remains recovered, indicates that grain foods were consumed" (Wendorf and Schild 2001, p. 590 et 671). Les documents disponibles ne permettent pas d'envisager une production végétale (pas de céréales cultivées attestées).

8 L'Ibéromaurusien* de la grotte de Taforalt* (Maroc oriental) n'a livré qu'une seule meule (?) entière, provenant du niveau supérieur IV; il s'agit d'une plaque rectangulaire $(\mathrm{L} .23 \mathrm{~cm})$ dont la surface lisse est ornée, à une extrémité, d'une paire de demi-cercles alignés côte à côte, incisés, suggestifs du cornage d'un mouflon (Roche 1958-1959, p. 183, pl. II). Aucune autre véritable meule entière n’a été signalée; les fragments analysés restent souvent muets et leurs dimensions insuffisantes n'évoquent pas les conditions dynamiques d'une activité de broyage exigeante. Dans la grotte 
d'Afalou* (Babors, Algérie), émergent d'une profusion de fragments, molettes, palets ou disques, entiers et typés, porteurs de marques suggestives; la molette ibéromaurusienne, caractéristique dans sa forme acquise (section plan-convexe), l'est plus encore en raison des zones d'usure des faces et du chant, qu'elle conserve finement piquetés, et surtout du colorant rouge resté intimement fixé (fonction symbolique). Les matériaux lithiques constitutifs furent à dessein choisis, il ne s'agit pas d'une variabilité de hasard. Dans la couche IV MI E1, deux documents purent être qualifiés de petites meules (Hachi 1997, 2003a).

Durant le Capsien, on a souvent relevé la présence de pièces de broyage entières (Balout 1955), pour la plupart des molettes, n'ayant pas échappé à la vigilance des pionniers, bien que mêlées aux nombreuses autres pierres de foyers. A Medjez II*, cet équipement découvert en maints endroits stratigraphiquement définis se rattache au faciès Sétifien* du Capsien supérieur (Camps-Fabrer 1975). Les meules ne répondent pas toutes à la diagnose ci-dessus, ce sont des témoins d'activités occasionnelles et intentionnelles, en raison des traces qu'ils portent. Petites dalles à angles arrondis et blocs naturels lourds, ne paraissant pas aptes à de longues activités réitérées. Dans le dépôt archéologique supérieur allant jusqu'à $0,25 \mathrm{~m}$ de profondeur, une dalle fut mise au jour portant quelques entailles balafrant l'une des faces (Camps-Fabrer 1975, p. 90, Fig. 37), cet exemple évoquerait plutôt l'utilisation d'un billot, destiné à découper ; à $0,75 \mathrm{~m}$ de profondeur, un autre bloc fut aussi considéré comme meule. Ailleurs (fouilles 1967-1968, A3, 1,25-1,50 m), l'auteur nous rend témoin de la découverte in situ d'une petite meule conservant sa molette, tachée d'ocre jaune, encore calée dans la concavité de la meule, créée par l'usage (Camps-Fabrer 1975, p. 114, Fig. 45). Cet excellent couple documentaire apporte la preuve de l'existence d'un équipement apparié (daté entre 7548-6837 cal BC, Cf. Tabl. 1 de Medjez II*), d'une dernière fonction de broyage de colorant, enfin d'un usage prolongé de ce couple d'objets (concavité). Les autres pièces représentent des meules primitives d'appoint. On conservera pourtant l'usage du terme «meule " comme un générique désignant tout support supposé remplir ces activités. Les caractéristiques des molettes capsiennes sont celles d'outils simples, rarement doubles (section bi-convexe), utilisés de chant. Leurs zones d'usure sont étendues à la face ou/et à la tranche, qui restent piquetées. Des colorants sont présents de façon étendue, superficielle, ou en épaisseur. Les poudres rouge et jaune dominent, comme sur certains outils lithiques taillés "Ces stigmates convergent vers une même pensée, celle de la prophylaxie dont on entourait les choses pour les rendre efficaces, mais invulnérables » (Camps-Fabrer 1975, p. 130). On retiendra que l'une des fonctions dominantes des molettes de Medjez II fut de livrer, après concassage et broyage, d'importantes productions poudreuses de substances colorantes. Cette activité fut mise en relation avec une situation à caractère symbolique (outils de cérémonie), comme l'accomplissement d'un cérémoniel funèbre lié à l'ensevelissement des défunts. Rappelons que Medjez II servit de nécropole aux individus du Sétifien du Constantinois.

Dans quelques escargotières du Capsien supérieur de Tébessa, d'autres documents ne sont pas des meules (Balout 1955). R. le Dû découvrit à l'Aïn Bahir une plaque couverte de colorants (Le Dû 1934), R. Vaufrey (1936-37) recueillit au Khanguet el-Mouhâad* une dalle portant de fines gravures ocrées, déchiffrées par H. Camps-Fabrer (1966, p. 214), d'autres "palettes à fard» proviennent du site de l'Aïn Rhilane (Vaufrey 1933). Cette documentation ayant joué un rôle de support de produits broyés constitue un autre ensemble à vocation symbolique. 
11 La Néolithisation du Maroc septentrional, récemment appréhendée dans la péninsule tingitane dans une perspective paléo-environnementale, est attestée, même si elle se perçoit moins bien à travers son matériel lithique, encore à l'étude. Aussi citerons-nous ici les nouveaux résultats palynologiques et carpologiques impliquant l'hypothèse du broyage, et faisant état de l'identification de céréales domestiques (probablement introduites d'Espagne). Il s'agit de graines carbonisées de blé dur et du blé amidonnier (Triticum dicoccum, T. aestivum/durum), mises en évidence dans des niveaux du Néolithique ancien Cardial, à Kaf Taht el-Ghar, vers la fin du $\mathrm{VI}^{\mathrm{e}}$ millénaire $\mathrm{BC}$ (Ballouche et Marinval 2003). On saluera la première information majeure sur ce sujet, obtenue pour le Maghreb méditerranéen.

La Néolithisation du faciès pastoral des régions atlasiques est aussi attestée. Elle puise dans l'environnement des matériaux pour façonner ses instruments de broyage (plaques de grés clivés, poudingues, quartzite, roches volcaniques) en meules simples, concaves, ou doubles à petites cuvettes, tandis que godets et mortiers sont rares. Au Damous el-Ahmar, ${ }^{*}$ aux environs de Tébessa (Roubet 1968, 1979, p.79-81), cet équipement s'est révélé assez abondant, diversifié et typé, mais les macro-restes végétaux n'ont pas été récoltés en 1912. Une palette à fard encore très chargée de colorants rouges, blanc et noir fut aussi recueillie en 1912 (Latapie in Roubet 1968), ainsi que plusieurs molettes ocrées (fonction symbolique). Dans la grotte Capéletti du Khanguet* Si Mohamed Tahar l'Aurès, trois séries d'occupations renfermaient des documents de broyage (Roubet 1979, Bachir-Bacha 1996). Le niveau supérieur livra in situ un couple d'outils appariés et typiques d'une meule et d'une molette (Roubet 1979, p. 148, Fig. 61); la fonction alimentaire de ce mobilier fut retenue et reliée à la découverte de glands doux du chêne ballotte, apparus de façon synchrone dans les dépôts des trois dernières séries d'occupation depuis 5207-4456 cal BC jusqu'à 3621-2471 cal BC (1). Il ne s'agit encore que de collectes sélectives, saisonnières. C. Roubet en déduisit une très vraisemblable production de semoule, de type grossier, pour une consommation de bouillies. En laboratoire, une expérimentation de broyage fut conduite avec les outils du site (op. cit., p. 442, Fig. 201), et des glands doux récoltés en automne, en montagne. Le résultat obtenu permit d'appuyer l'hypothèse d'un réel appoint alimentaire farineux, saisonnier. La définition de ce faciès atlasique pastoral du Néolithique de tradition capsienne ne fut complète que lorsqu'on admit de lui reconnaître un stade pré-agricole. Depuis cette information rien n'a changé dans la documentation algérienne rattachée à cette période.

On peut penser que ce comportement pré-agricole s'est étendu aux grottes de Brézina, de l'ouest pré-saharien de l'Atlas, où se développa un faciès pratiquant le broyage de graines sauvages (Delmas 1910). L'important équipement conservé à l'Institut de Paléontologie Humaine de Paris est rarement ocré (Illoul 2004). Tandis qu'en bordure des Chotts, en raison d'une intense déflation, les sites de plein air du Bas-Sahara oriental, pourvus en pièces lithiques, ne conservent presque plus de colorants et manquent de macro-restes végétaux et de témoins osseux (Aumassip 1986).

La Néolithisation ancienne du faciès saharo-soudanais, telle qu'Amekni* et Méniet (Hugot 1963) la représentent, est attestée; ce contexte comprend un abondant équipement de broyage simple. Si les meules sont présentes en surface, aucune ne fut découverte dans les sédiments d'Amekni, alors que les molettes y étaient nombreuses. En revanche plusieurs cuvettes ovalaires, profondes, creusées dans les masses granitiques environnantes, révélèrent «l'importance du travail de meunerie » (Camps 
1968, p. 99) exercé sur des graines d'un mil domestique*. La vocation alimentaire de cet équipement est directement confirmée. Même si rien encore ne permet d'assurer la contemporanéité de ces cuvettes avec l'occupation du site et la production de semoulefarine de mil.

Au Sud du Tassili n'Ajjer, dans l'erg d'Admer occupé par le faciès septentrional du Néolithique pastoral (?) du "Ténéréen » (centré au Niger, avec l'Adrar Bous comme référence), un équipement de meunerie très caractéristique paraît avoir été développé et abondant. Il s'agit de meules transportables quoique lourdes, largement ovalaires ou circulaires, très élaborées, dont la morphologie finale résulte d'aménagements appropriés assurant la stabilité, la préparation du rebord, la création d'un marli et même parfois la décoration. Après usage, les molettes ont acquis divers caractères particuliers (carènes, gouttières, cupules), révélant la complexité et la multiplicité des fonctions. La production de poudres colorantes ne parait pas conservée, les sites de plein air ont perdu leur contexte botanique qui aurait donné sens et vie à cet équipement. On ne conteste pas à cet équipement une vocation alimentaire qui dut rester occasionnelle et dépendante de collectes et que conforte aussi la vaisselle céramique hémisphérique (Camps 1974). Mais, pour l'instant, C. Roubet considère le Ténéréen comme n'ayant pas dépassé un stade pré-agricole.

En Libye, en territoire saharien, les données obtenues par des équipes multidisciplinaires étudiant le massif de l'Acacus durant l'Holocène, insistent sur la durée, la continuité des occupations pré-pastorales et la précocité pastorale (fin du VIII ${ }^{e}$ millénaire BP, non calibré). Le mobilier de plusieurs contextes culturels successifs comprend toujours des instruments de broyage. Les macro-restes végétaux de la période pastorale restent encore des «wild plant species» reflétant des activités constantes, prédatrices et sélectives. E. Garcea (2004) précise à propos de la présence de Boraginaceae et Urochloa «These plants could grow or increase their production in certain ecological niches only thanks to human skills » (Mercuri 1999, 2001 ; Mercuri and Garcea 2003).

Dans l'état actuel de nos connaissances, l'équipement de broyage fait partie depuis longtemps du mobilier préhistorique d'Homo sapiens sapiens, qui l'a largement exploité à des fins symboliques et pré-agricoles. On ne saurait le dissocier de ses multiples fonctions, directement ou indirectement attestées. Mais à l'exception du mil d'Amekni, du blé amidonnier et du blé dur de Kaf Taht el-Ghar, aucune autre production alimentaire végétale, résultant d'une mise en culture, n'est attestée à ce jour au Maghreb et au Sahara, durant l'Holocène.

\section{BIBLIOGRAPHIE}

AUMASSIP G., 1986 - Le Bas-Sahara dans la Préhistoire, Paris, CNRS, Etudes d'Antiquités africaines. 
BACHIR-BACHA A., 1996 - Réexamen du Néolithique de tradition capsienne, d'apres le matériel de la grotte Capéletti (Algérie Orientale), thèse de Doctorat de l'Université de Poitiers, Département d'Histoire et d'Archéologie.

BALLOUCHE A., et MARINVAL Ph., 2003 - « Données palynologiques et carpologiques sur la domestication des plantes et l'agriculture dans le Néolithique ancien du Maroc septentrional (site de Kaf Taht el-Ghar) », Revue d'Archéométrie, t. 27, p. 49-54.

BALOUT L., 1955 - Préhistoire de l'Afrique du Nord. Essai de chronologie, Paris, AMG.

CAMPS G., 1968 - Amekni, Néolithique ancien du Hoggar, Mémoire X du CRAPE Paris, AMG.

CAMPS G., 1974 - Les civilisations préhistoriques de l'Afrique du Nord et du Sahara, Paris, Doin.

CAMPS-FABRER H., 1966 - Matière et art mobilier dans la Préhistoire nord-africaine et saharienne, Paris, AMG, Mémoire V du CRAPE.

CAMPS-FABRER H., 1975 - Un gisement Capsien de faciès Sétifien : Medjez II. El Eulma (Algérie), Paris, CNRS, Etudes d'Antiquités Africaines. Avec la collab. de J. Bouchud, M. Chabeuf, M.-C. Chamla, M. Couvert, R. Dughi, et F. Sirugue.

DELMAS M.-P., 1910 - « Notes sur les grottes de Brézina. Contribution à l'étude archéologique préhistorique dans l'Afrique du Nord », XXXIXe Congrès de l'AFAS, Toulouse, p. 367-379.

GARCEA E. A.-A., 2004 - "An alternative way towards food production : the perspective from the Libyan Sahara", Journal of World Prehistory, vol. 18, n² 2, p. 107-154.

HACHI S., 1999 - L'Homme de Mechta-Afalou, Cro-Magnon de l'Afrique du Nord durant les 20 derniers millénaires : évolution culturelle et devenir, Thèse d'Habilitation à Diriger des Recherches. Université Paul Valéry, Montpellier III, 1999, 4 volumes.

HACHI S., 2003 - Les cultures de l'Homme de Mechta-Afalou. Le gisement d'Afalou Bou Rhummel (massif des Babors, Algérie). Les niveaux supérieurs 13.000-11.000 BP, Alger, CNRPAH.

HUGOT H.-J., 1963 - Recherches préhistoriques dans l'Ahaggar nord-occidental. 1950-1957, Paris, AMG, Mémoire I du CRAPE.

ILloUl K., 2004 - « Réévaluation des collections néolithiques des grottes d'El Arouia, prés de Brézina (Atlas saharien), Algérie », Mémoire de D.E.A., Quaternaire et Préhistoire du MNHN-IPH, Paris.

LE DÛ R., 1934 - « Une station capsienne. L'escargotière d'Aïn Bahir », Recueil des Notices et Mémoires de la Société Archéol. de Constantine, t. LXII, p. 81-90.

MERCURI A.-M., 1999 - "Palynological analysis of the Early Holocene Sequence" in di Lernia, S. (eds.). The Uan Afuda Cave : hunter gatherer societies of Central Sahara, Firenze, All'Insegna del Giglio, p. $149-181$.

MERCURI A.-M., 2001 - "Preliminary analyses of fruits and seeds from the Early Holocene Sequence", in Garcea E. A.-A., (ed.), Uan Tabu in the settlement history of the Libyan Sahara. Firenze, All'Insegna del Giglio, p. 189-210.

MERCURI A.-M. and GARCEA E. A.-A., 2003 - "Plant exploitation and landscape at Uan Tabu-Tadrart Acacus : Hunter-gatherers and plant world in the Central Sahara during the Early Holocene", Paper presented at the $4^{\text {th }}$ International Workshop on African Archaeobotany. Groningen Archaeological Studies, Groningen, Barkhuis Publishing and Groningen University Library. ROCHE J., 1958-1959 - « L'Epipaléolithique marocain », Libyca, t. VI-VII, p. 159-192. 
ROUBET C., 1968 - Le gisement du Damous el Ahmar et sa place dans le Néolithique de tradition capsienne ", Paris, AMG (Travaux du CRAPE).

ROUBET C., 1973 - « Essai de classification du matériel de broyage », Actes du VIII ${ }^{e}$ Congrès UISPP, Belgrade, t. 3, p. 413-417.

ROUBET C., 1979 - Economie pastorale préagricole en Algérie orientale. Le Néolithique de tradition capsienne. Exemple l'Aurès, CNRS (Etudes d'Antiquités Africaines).

ROUBET C., 1989a - "The grinding stones of site E. 78-3 at Wadi Kubbaniya", The Prehistory of Wadi Kubbaniya. Late Palaeolithic Archaeology. Assembled by F. Wendorf and R. Schild, ed. by A. Close, Southern Methodist University, vol. 3, p. 473-489.

ROUBET C., 1989b - "Report on site E.82-1 : a workshop for manufacture of grinding stones at Wadi Kubbaniya", Prehistory of Wadi Kubbaniya. Late Palaeolithic Archaeology. Assembled by F. Wendorf and R. Schild, ed. by A. Close, Southern Methodist University, vol. 3, p. 588-608.

VAUfREY R., 1933 - « Notes sur le Capsien », L’Anthropologie, t. XLIII, p. 457-483.

VAUfRey R., 1936-1937 - « Le Capsien des environs de Tébessa », Bull, de la Société de Préhistoire et d'Archéologie de Tébessa, t.1, p. 131-172.

VAUFREY R., 1939 - L'art rupestre nord-africain. Paris, Masson, Archives de l'IPH, Mémoire XX.

WENDORF F. and SCHILD R., 2001 - Holocene settlement of the Egyptian Sahara. Vol. 1. The Archaeology of Nabta Playa. Kluwer Academic / Plenum Publishers.

\section{INDEX}

Mots-clés : Agriculture, Alimentation, Ethnologie, Ethnographie, Néolithique, Préhistoire, Technologie 